\title{
Total Flavonoid Content, the Antioxidant Capacity, Fingerprinting and Quantitative Analysis of Fupenzi (Rubus chingii Hu.)
}

\author{
Huajin Zeng', Ran Yang2 ${ }^{*}$, Lifang Lei ${ }^{2}$, Yaping Wang1 \\ ${ }^{1}$ School of Pharmaceutical Sciences, Zhengzhou University, Zhengzhou, China \\ ${ }^{2}$ College of Chemistry and Molecular Engineering, Zhengzhou University, Zhengzhou, China \\ Email: ${ }^{2}$ yangran@zzu.edu.cn
}

Received 2 November 2015; accepted 28 December 2015; published 31 December 2015

Copyright (C) 2015 by authors and Scientific Research Publishing Inc.

This work is licensed under the Creative Commons Attribution International License (CC BY). http://creativecommons.org/licenses/by/4.0/

(c) (i) Open Access

\section{Abstract}

Objective: In this study, one of the objectives was to investigate the total flavonoid contents of Fupenzi ( $R$. chingii Hu.) obtained from different regions of China and to evaluate their anatioxidant activities. And the second objective of this study was to develop a validated HPLC method for chromatographic fingerprints of the samples extracts of Fupenzi. Method: The total flavonoid contents were determined by a colorimetric method and the antioxidant activity was determined spectrophotometrically by DPPH and ABTS radical scavenging assays. The chromatographic fingerprint was developed by high-performance liquid chromatography coupled with diode array detection for the control of Fupenzi. Results: A significant correlation between antioxidant activity and the total flavonoid content was observed for the DPPH assay $\left(r^{2}=0.758, \rho=0.004\right)$ and the ABTS assay $\left(r^{2}=0.788, \rho=0.002\right)$. Under the optimized chromatographic conditions, the validated method was successfully applied to assessment of chemical fingerprinting of 12 batches of FPZ collected from different regions of China. Comparisons of the chromatograms showed that 15 characteristic peaks could be selected as markers for identification and evaluation of Fupenzi. In addition, the proposed method was also successfully applied to simultaneous determination of five compounds (including puerarin, rutin, hyperin, quercetin and kaempferol) in these samples. Conclusions: The qualitative and quantitative analysis described in this paper could be used for identification and evaluation of Fupenzi.

\section{Keywords}

Flavonoid, Antioxidant Capacity, Fingerprinting, Quantitative Analysis, Fupenzi (Rubus chingii Hu.)

\footnotetext{
${ }^{*}$ Corresponding author.
} 


\section{Introduction}

As a member of the Rosaceae family grown as a perennial crop, raspberry is widely grown in China and is popular for its flavour and attractive red color. In addition to being a favorite food, its unripe fruits are usually used as a key member of many ancient prescriptions against aging [1]. Modern pharmacological research showed that scavenging free radicals in the body by natural antioxidants was an effective way for anti-aging. Berries, such as blackberry, raspberry and strawberry, contain high levels of anthocyanins, flavonoids and phenolic acids and are considered a good source of natural antioxidant [2] [3], which might provided protection against various human disease caused by oxidative stress. Therefore, it is very significant to evaluate the antioxidant activity of different kinds of berries. However, to the best of our knowledge, there is no report on evaluation of the antioxidant capacity of Fupenzi (R. chingii Hu.).

Since the entire pattern of compounds characterizes the chemical composition of the herbs, the chromatographic fingerprint represents a comprehensive qualitative methodology, in which the entire chromatogram is evaluated during data analysis to discriminate between different species of the same herbal family [4]-[7]. Nowadays, chemical fingerprinting has been internationally accepted as an efficient technique for the assessment of not only herbal medicines, but also botanical food, including the leaves of $R$ suavissimus S. Lee [8] and the fruits of $R$. chingii Hu. [9]. However, there were not enough common peaks (only 7 common peaks) selected as characteristic peaks for the identification of the fruits of $R$. chingii Hu. and there was lack of the combination of the constituents contributed to antioxidant activity with chemical fingerprinting.

Taking all these factors into considerations, the objective of this study was to investigate the total flavonoid contents of Fupenzi obtained from different regions of China and to evaluate their antioxidant activities. And the second objective of this study was to develop a validated HPLC method for chromatographic fingerprints of the sample extracts of Fupenzi.

\section{Experimental}

\subsection{Chemicals and Reagents}

Twelve batches of Fupenzi samples were collected from different regions of China (1-Hangzhou (Zhejiang), 2-Jinhua (Zhejiang), 3-Zhengzhou (Henna), 4-Nanjing (Jiangsu), 5-Guangzhou (Guangdong), 6-Guilin (Guangxi), 7-Nanning (Guangxi), 8-Chengdu (Sichuang), 9-Fuzhou (Fujian), 10-Zhangzhou (Fujian), 11-Changchun (Jilin), 12-shengyang (Jilin)). Rutin, hyperin, quercetin and kaempferol were purchased from National Institute for the Control of Pharmaceutical and Biological Products (Beijing, China). DPPH (2,2-diphenyl-1-picrylhydrazyl) and ABTS (2,2'-azinobis[3-ethylbenzothiazoline-6-sulphonic acid] diammonium salt) were purchased from Sigma chemical Co. (Sigma, USA). Acetonitrile and methanol were of HPLC grade (Shield Fine Chemicals Company, Tianjin, China). Water for HPLC analysis was purified by a Milli-Q water purification system (Shanghai, China). Other reagents were all of analytical grade.

\subsection{Preparation of Sample Solutions}

Grind the fruit samples of Fupenzi into powder by use of a pestle and mortar. $3.0 \mathrm{~g}$ pulverized powder was accurately weighed and ultrasonically extracted with $40 \mathrm{~mL}$ of $95 \%$ ethanol for 50 min in a calibrated flask at $40^{\circ} \mathrm{C}$, and then filtered through a $0.22 \mu \mathrm{m}$ membrane. The filtrate was evaporated to dryness at $35^{\circ} \mathrm{C}$ and the residue was dissolved into $10 \mathrm{~mL}$ methanol. The solution was filtrated through a syringe filter $(0.22 \mu \mathrm{m})$ and aliquots $(20 \mu \mathrm{L})$ were subjected to HPLC before analysis.

\subsection{Preparation of Standard Solutions}

A standard stock solution containing the five components (rutin, hyperin, quercetin and kaempferol) was prepared in methanol and stored away from light at $4^{\circ} \mathrm{C}$. Working standard solutions containing the four compounds were prepared by appropriate dilution of the stock solution.

\subsection{Determination of the Total Flavonoid Content}

The total flavonoid content (TFC) was determined using a colorimetric method [10] with a little modification. $0.1 \mathrm{~mL}$ properly diluted sample of the extract was mixed with $0.3 \mathrm{~mL}$ of a $5 \% \mathrm{NaNO}_{2}$ solution. The mixture was 
allowed to stay at room temperature for $6 \mathrm{~min} ; 0.3 \mathrm{~mL}$ of a $10 \% \mathrm{AlCl}_{3}$ solution was added for 6 min followed by the addition of a $2 \mathrm{~mL} 4 \% \mathrm{NaOH}$ solution. The double-distilled water was added to reach a final volume of 10 $\mathrm{ml}$. The solution was mixed and kept at room temperature for $15 \mathrm{~min}$. Absorbance was measured immediately against the prepared blank at $510 \mathrm{~nm}$ using a spectrophotometer (UV-2550, Shimadzu, equipped with $10 \mathrm{~mm}$ quartz cuvettes). Quantitative measurements were performed, based on a standard calibration curve of six points from 5.0 to $80 \mathrm{mg} \cdot \mathrm{L}^{-1}$ of rutin in methanol $\left(y=0.0105 x-0.0031, \mathrm{R}^{2}=0.9965, y\right.$ and $x$ represent the absorbance and concentration, respectively). The total phenolic content was expressed as rutin equivalents in $\mathrm{mg} \cdot \mathrm{g}^{-1}$ of extract.

\subsection{DPPH Assay}

DPPH radical-scavenging capacity of raspberry extracts was evaluated according to the method of Chen and Ho [11], with a little modification. Briefly, $0.05 \mathrm{~mL}$ properly diluted sample of the extract was added to $5.0 \mathrm{~mL}$ methanol solution of DPPH radical (final concentration was $0.5 \mathrm{mmol} \cdot \mathrm{L}^{-1}$ ). The mixture was shaken vigorously for $1 \mathrm{~min}$ by vortexing and left to stand at room temperature in the dark for $30 \mathrm{~min}$. Thereafter, the absorbance of the sample was measured using the UV-vis spectrophotometer at $517 \mathrm{~nm}$ against methanol blank. A negative control was taken after adding DPPH solution to $0.05 \mathrm{~mL}$ methanol. The ascorbic acid was used as a reference compound $\left[y=14.032 x+0.4534, \mathrm{R}^{2}=0.9975, y\right.$ and $x$ represent the scavenging rate and concentration $(0.006$ $5.6 \mathrm{mg} \cdot \mathrm{L}^{-1}$ ), respectively]. The ascorbic acid equivalent antioxidant capacity was expressed as $\mathrm{mg}$ ascorbic acid corresponding to one g dry weight.

\subsection{ABTS Assay}

The ABTS radical scavenging test was used to determine the antioxidant activity [12]. ABTS radical was obtained by reaction between ABTS and potassium persulfate. Blank sample was prepared from the daily solution by adding $100 \mathrm{~mL}$ PBS buffer ( $5 \mathrm{mmol} \cdot \mathrm{L}^{-1}$, $\mathrm{pH} 7.4$ ), which gives an absorbance of $0.7 \pm 0.02$. The radical scavenging activity was assessed by mixing $5.0 \mathrm{~mL}$ ABTS solution with $0.05 \mathrm{~mL}$ properly diluted sample of the extract. The reactive mixture was allowed to stand at room temperature for $6 \mathrm{~min}$ and the absorbance was recorded at $734 \mathrm{~nm}$. The ascorbic acid was used as standard $\left(y=22.821 x+1.3393, \mathrm{R}^{2}=0.9978, y\right.$ and $x$ represent the scavenging rate and concentration $\left(0.005-4.0 \mathrm{mg} \cdot \mathrm{L}^{-1}\right)$, respectively). The ascorbic acid equivalent antioxidant capacity was calculated like DPPH assay.

\subsection{HPLC Conditions}

The HPLC system Dionex P680 series (Dionex, USA), equipped with the Chromeleon software (Dionex) and comprised a binary pump, an online vacuum degasser, a manual sampler, a thermostated column compartment and a diode array detection (DAD), was used for the chromatographic analysis. The chromatographic analysis was carried out on a Kromasil $\mathrm{C}_{18}(250 \mathrm{~mm} \times 4.6 \mathrm{~mm}, 5.0 \mu \mathrm{m})$ column. The mobile phase was composed of acetonitrile (A) and 1\% formic acid solution (B) with gradient elution (0 - 5 min, 5\% - 20\% A; 5 - 20 min, 20\% 45\% A; 20 - 25 min, 45\% - 90\% A; 25 - 30 min, 90\% - 100\% A, 30 - 40 min, 100\% A). The solvent flow rate was $1.0 \mathrm{~mL} \cdot \mathrm{min}^{-1}$, the elute was continuously monitored using a DAD detector at a wavelength of $360 \mathrm{~nm}$ and the column temperature was maintained at $30^{\circ} \mathrm{C}$. Aliquots of $20 \mu \mathrm{L}$ were injected into HPLC system for analysis.

\subsection{Statistical Analysis}

Data analysis was performed using professional software (Analysis System for Chromatographic Fingerprint and Data of Traditional Chinese Medicine version 2004, the Pharmacopoeia Commission of the People's Republic of China). This software allowed calculation of the correlation coefficients of the sample from different technologies with the reference chromatogram. The hierarchical clustering analysis (HCA) and principal component analysis (PCA) was performed using SPSS for Windows v.18.0 software (SPSS Corporation, Stanford, USA).

\section{Results and Discussion}

\subsection{Total Flavonoid Content, DPPH and ABTS Radical Scavenging Activities}

The content of total flavonoid was determined by a colorimetric method and the results were summarized in Ta- 
ble 1. The total flavonoid contents in all samples ranged from $13.17 \pm 0.02$ to $22.82 \pm 0.02 \mathrm{mg}$ rutin $\mathrm{g}^{-1}$ d.w. The values for DPPH and ABTS scavenging activities in all samples ranged from $20.51 \pm 0.06$ to $31.08 \pm 0.03$ $\mathrm{mg}$ ascorbic acid $\mathrm{g}^{-1}$ d.w. and from $33.93 \pm 0.11$ to $54.83 \pm 0.12 \mathrm{mg}$ ascorbic acid $\mathrm{g}^{-1}$ d.w., respectively (Table 1). A significant correlation between DPPH assay and ABTS assay was observed $\left(r^{2}=0.821, \rho=0.001\right.$, SPSS for windows 19.0, USA), which suggested the two methods were comparable in the case of Fupenzi (Table 2).

Generally, the total flavonoid contents were positively correlated with the antioxidant activities [13] [14]. In this study a significant correlation between antioxidant activity and the total flavonoid content was also observed for the DPPH assay $\left(r^{2}=0.758, \rho=0.004\right)$ and the ABTS assay $\left(r^{2}=0.788, \rho=0.002\right)$ (Table 2).

\subsection{HPLC Chromatograms of Fupenzi}

With the same sample extracts contributed to antioxidant activities, the HPLC chromatograms of 12 batches of samples were obtained under the optimized chromatographic conditions (Figure 1). As shown in Figure 1, these samples showed similar chromatographic profiles and more than 22 peaks were detected in all the 12 chromatograms. After carefully analyzing the chromatographic profiles of these samples, 15 common peaks with acceptable heights and good resolution were selected as characteristic peaks for fingerprinting analysis (Figure 2). Peak 10 was selected as the reference peak. Relative retention times (RRTs) and relative peak areas (RPAs) of the 15 characteristic peaks were calculated as follows: RRT = retention time of characteristic peak/retention time of marker peak, and RPA = peak area of characteristic peak/peak area of marker peak. The results from the 12 samples indicated that the RPAs of the 15 characteristic peaks varied dramatically (Table 3), but the RRTs were invariable for the Fupenzi (Table 4). The results indicated that the RRTs of the 15 characteristic peaks can serve as characteristic peaks for identification of "unknown" samples. A sample with a similar HPLC chromatographic profile and matched RRT values to the typical fingerprint chromatogram can be authenticated as genuine Fupenzi. However, due to several ingredients, such as climate, season of harvest and regions of cultivation, the same types of components were generally existed in the same variety, but their contents were often different

Table 1. The total flavonoid contents, DPPH and ABTS scavenging activities in the sample extract of 12 raspberries ( $R$. chingii Hu.). $(\mathrm{n}=6)$.

\begin{tabular}{cccc}
\hline Samples & $\begin{array}{c}\text { Total flavonoids content } \\
\text { (mg rutin }{ }^{-1} \text { d.w.) }\end{array}$ & $\begin{array}{c}\text { DPPH } \\
\text { (mg ascorbic acid } \mathrm{g}^{-1} \text { d.w.) }\end{array}$ & $\begin{array}{c}\text { ABTS } \\
\text { (mg ascorbic acid g d.w.) }\end{array}$ \\
\hline Hangzhou & $14.87 \pm 0.02$ & $20.51 \pm 0.06$ & $33.93 \pm 0.11$ \\
Jinhua & $17.08 \pm 0.03$ & $25.65 \pm 0.03$ & $48.67 \pm 0.06$ \\
Zhengzhou & $19.50 \pm 0.03$ & $31.08 \pm 0.03$ & $54.26 \pm 0.12$ \\
Nanjing & $17.40 \pm 0.03$ & $27.01 \pm 0.03$ & $47.86 \pm 0.07$ \\
Guangzhou & $17.50 \pm 0.03$ & $24.68 \pm 0.05$ & $42.93 \pm 0.06$ \\
Guilin & $19.60 \pm 0.02$ & $25.94 \pm 0.05$ & $41.62 \pm 0.12$ \\
Nanning & $13.17 \pm 0.02$ & $21.98 \pm 0.03$ & $37.99 \pm 0.07$ \\
Chengdu & $19.16 \pm 0.02$ & $26.70 \pm 0.05$ & $49.51 \pm 0.06$ \\
Fuzhou & $18.27 \pm 0.02$ & $27.97 \pm 0.03$ & $42.05 \pm 0.06$ \\
Zhangzhou & $15.52 \pm 0.05$ & $21.69 \pm 0.05$ & $37.41 \pm 0.07$ \\
Changchun & $22.82 \pm 0.02$ & $26.90 \pm 0.06$ & $54.83 \pm 0.12$ \\
Shenyang & $14.88 \pm 0.02$ & $21.11 \pm 0.08$ & $40.55 \pm 0.13$ \\
\hline
\end{tabular}

Table 2. Pair-wise correlations among total flavonoid content, DPPH and ABTS.

\begin{tabular}{cll}
\hline & DPPH & ABTS \\
\hline Total flavonoid content & $0.758^{*}$ & $0.788^{*}$ \\
DPPH & & $0.821^{*}$ \\
\hline
\end{tabular}

*Was significant at 0.01 probability level. 
Table 3. Relative peak areas (RPAs) of 15 characteristic peaks in HPLC fingerprints of 12 samples of FPZs.

\begin{tabular}{|c|c|c|c|c|c|c|c|c|c|c|c|c|c|c|c|}
\hline \multirow[t]{2}{*}{ Samples } & \multicolumn{15}{|c|}{ Peaks } \\
\hline & 1 & 2 & 3 & 4 & 5 & 6 & 7 & 8 & 9 & 10 & 11 & 12 & 13 & 14 & 15 \\
\hline 1 & 3.921 & 1.579 & 1.500 & 1.303 & 1.789 & 41.961 & 3.711 & 2.711 & 0.224 & 1.000 & 0.553 & 1.921 & 2.474 & 5.513 & 0.645 \\
\hline 2 & 2.421 & 0.871 & 1.374 & 0.807 & 2.158 & 30.474 & 4.965 & 3.760 & 0.193 & 1.000 & 0.327 & 0.620 & 0.854 & 3.222 & 0.304 \\
\hline 3 & 5.307 & 1.973 & 4.347 & 3.000 & 5.520 & 66.107 & 8.347 & 3.640 & 0.107 & 1.000 & 0.360 & 1.293 & 1.400 & 4.253 & 0.387 \\
\hline 4 & 4.155 & 0.613 & 0.735 & 0.826 & 1.555 & 32.981 & 3.432 & 2.594 & 0.226 & 1.000 & 0.303 & 0.858 & 1.052 & 2.574 & 0.226 \\
\hline 5 & 2.514 & 1.140 & 0.444 & 0.720 & 0.252 & 29.591 & 0.633 & 0.962 & 0.227 & 1.000 & 0.066 & 0.266 & 0.451 & 1.066 & 0.014 \\
\hline 6 & 4.965 & 1.451 & 0.876 & 1.496 & 0.779 & 43.496 & 2.257 & 1.646 & 0.381 & 1.000 & 0.221 & 0.531 & 0.938 & 2.885 & 0.257 \\
\hline 7 & 4.289 & 1.505 & 1.011 & 0.847 & 1.242 & 39.211 & 3.326 & 1.868 & 0.332 & 1.000 & 0.295 & 0.679 & 0.868 & 2.337 & 0.189 \\
\hline 8 & 6.397 & 0.446 & 0.306 & 0.066 & 0.653 & 2.562 & 1.289 & 4.041 & 0.281 & 1.000 & 0.322 & 1.149 & 1.397 & 3.066 & 0.264 \\
\hline 9 & 3.249 & 0.633 & 1.096 & 0.847 & 2.667 & 31.701 & 3.808 & 2.322 & 0.175 & 1.000 & 0.424 & 1.153 & 1.345 & 3.520 & 0.333 \\
\hline 10 & 6.510 & 1.657 & 2.294 & 1.618 & 1.931 & 59.206 & 11.049 & 5.814 & 0.324 & 1.000 & 0.324 & 1.431 & 1.667 & 4.235 & 0.343 \\
\hline 11 & 0.267 & 1.168 & 0.168 & 1.534 & ------ & 69.351 & 0.603 & 0.588 & 0.672 & 1.000 & 0.214 & 0.550 & 1.015 & 3.115 & 0.252 \\
\hline 12 & 5.491 & 1.613 & 0.943 & 1.406 & 0.425 & 51.981 & 1.698 & 2.807 & 0.245 & 1.000 & 0.052 & 0.297 & 0.425 & 0.679 & 0.042 \\
\hline Mean & 4.124 & 1.221 & 1.258 & 1.206 & 1.581 & 41.552 & 3.760 & 2.729 & 0.282 & 1.000 & 0.288 & 0.896 & 1.157 & 3.039 & 0.271 \\
\hline RSD (\%) & 181.00 & 48.79 & 113.02 & 72.15 & 148.99 & 1849.35 & 313.75 & 144.42 & 14.36 & 0.00 & 13.91 & 50.08 & 55.88 & 133.11 & 16.27 \\
\hline
\end{tabular}

Table 4. Relative retention times (RRTs) of 15 characteristic peaks in HPLC fingerprints of 12 samples of FPZs.

\begin{tabular}{|c|c|c|c|c|c|c|c|c|c|c|c|c|c|c|c|}
\hline \multirow[t]{2}{*}{ Samples } & \multicolumn{15}{|c|}{ Peaks } \\
\hline & 1 & 2 & 3 & 4 & 5 & 6 & 7 & 8 & 9 & 10 & 11 & 12 & 13 & 14 & 15 \\
\hline 1 & 0.394 & 0.424 & 0.452 & 0.470 & 0.487 & 0.515 & 0.585 & 0.785 & 0.843 & 1.000 & 1.488 & 1.573 & 1.592 & 1.616 & 1.653 \\
\hline 2 & 0.396 & 0.425 & 0.453 & 0.472 & 0.488 & 0.515 & 0.586 & 0.786 & 0.843 & 1.000 & 1.489 & 1.574 & 1.596 & 1.616 & 1.654 \\
\hline 3 & 0.394 & 0.424 & 0.453 & 0.471 & 0.487 & 0.514 & 0.586 & 0.785 & 0.843 & 1.000 & 1.487 & 1.572 & 1.593 & 1.615 & 1.652 \\
\hline 4 & 0.394 & 0.423 & 0.453 & 0.471 & 0.488 & 0.514 & 0.586 & 0.786 & 0.843 & 1.000 & 1.489 & 1.574 & 1.596 & 1.618 & 1.654 \\
\hline 5 & 0.393 & 0.421 & 0.452 & 0.472 & 0.489 & 0.513 & 0.570 & 0.786 & 0.843 & 1.000 & 1.481 & 1.565 & 1.586 & 1.607 & 1.644 \\
\hline 6 & 0.396 & 0.424 & 0.454 & 0.472 & 0.489 & 0.515 & 0.587 & 0.786 & 0.844 & 1.000 & 1.486 & 1.570 & 1.591 & 1.612 & 1.650 \\
\hline 7 & 0.394 & 0.423 & 0.453 & 0.472 & 0.488 & 0.513 & 0.585 & 0.786 & 0.844 & 1.000 & 1.488 & 1.572 & 1.594 & 1.615 & 1.652 \\
\hline 8 & 0.394 & 0.423 & 0.452 & 0.459 & 0.470 & 0.487 & 0.567 & 0.785 & 0.843 & 1.000 & 1.492 & 1.576 & 1.597 & 1.619 & 1.656 \\
\hline 9 & 0.394 & 0.424 & 0.452 & 0.470 & 0.487 & 0.513 & 0.586 & 0.785 & 0.844 & 1.000 & 1.488 & 1.573 & 1.594 & 1.616 & 1.653 \\
\hline 10 & 0.396 & 0.425 & 0.454 & 0.471 & 0.488 & 0.514 & 0.592 & 0.785 & 0.843 & 1.000 & 1.491 & 1.576 & 1.598 & 1.619 & 1.657 \\
\hline 11 & 0.395 & 0.423 & 0.453 & 0.471 & ------ & 0.513 & 0.598 & 0.785 & 0.843 & 1.000 & 1.487 & 1.572 & 1.593 & 1.615 & 1.652 \\
\hline 12 & 0.395 & 0.423 & 0.452 & 0.470 & 0.487 & 0.510 & 0.584 & 0.785 & 0.843 & 1.000 & 1.492 & 1.577 & 1.598 & 1.619 & 1.657 \\
\hline Mean & 0.395 & 0.424 & 0.453 & 0.470 & 0.446 & 0.511 & 0.584 & 0.785 & 0.843 & 1.000 & 1.488 & 1.573 & 1.594 & 1.616 & 1.653 \\
\hline RSD (\%) & 0.10 & 0.11 & 0.08 & 0.36 & 0.14 & 0.78 & 0.84 & 0.05 & 0.05 & 0.00 & 0.30 & 0.32 & 0.34 & 0.34 & 0.35 \\
\hline
\end{tabular}

[15].

Due to similarity in appearance, it is impossible to divide these samples from the physical appearances. According to the RRTs and RPAs of 15 characteristic peaks, hierarchical clustering analysis (HCA) was perforemed by SPSS software (SPSS for windows 19.0, SPSS Inc., USA) and the results were shown in Figure 3 . It is clear that the samples could be divided into three clusters: with samples 1, 2, 4, 5, 6, 7 and 9 in cluster one, samples 3, 10, 11 and 12 in cluster two and sample 8 in cluster three. The rescaled distance between group three and group one, group two are 25, while the rescaled distance between group one and group two are 10, which could demonstrate that the quality of group one and group two are more similar than with group three. 


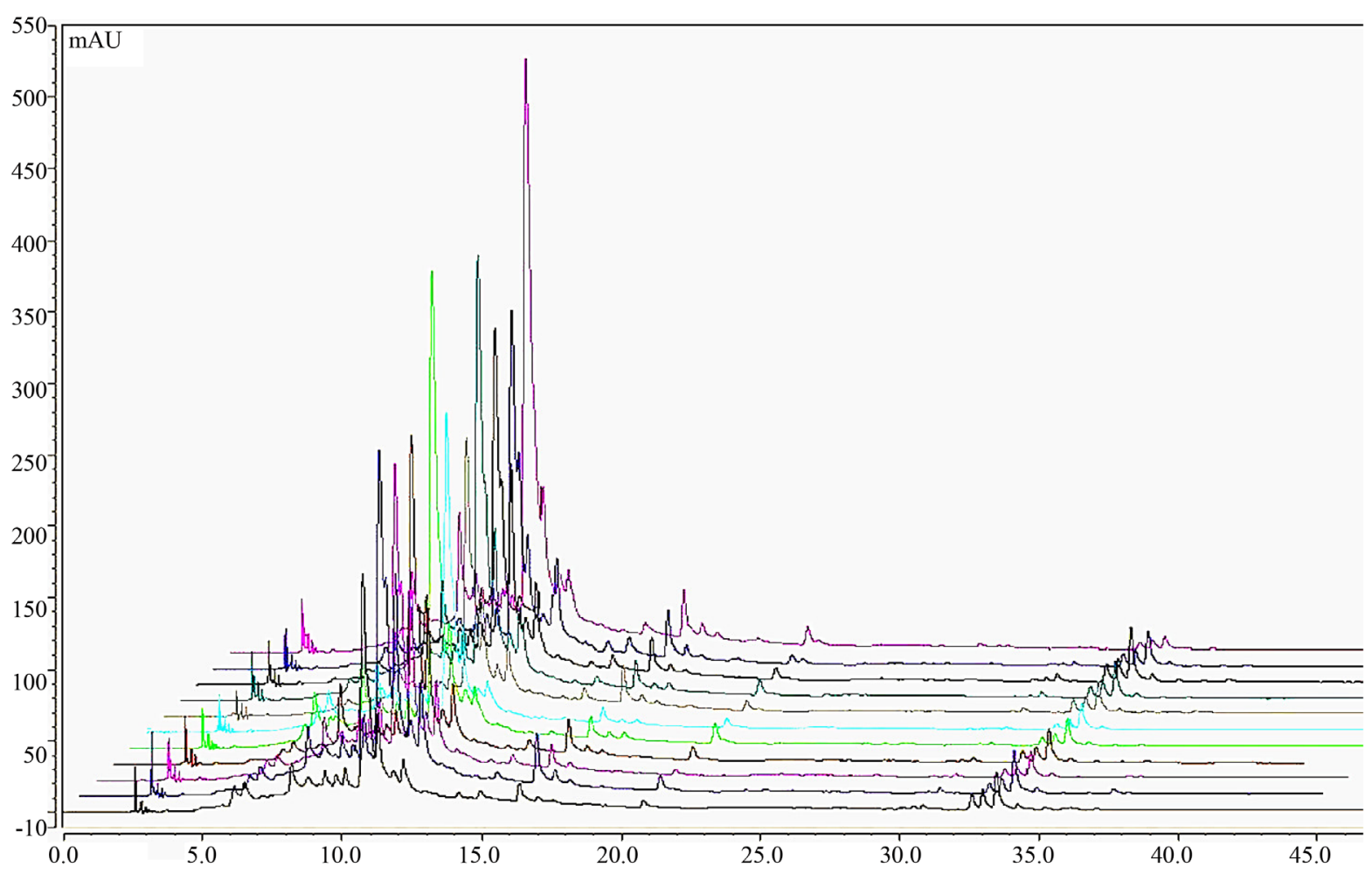

Figure 1. Overlaid HPLC chromatograms of extracts of Samples. From down to up: Hangzhou, Jinhua, Zhengzhou, Nanjing, Guangzhou, Guilin, Nanning, Chengdu, Fuzhou, Zhangzhou, Changchun and Shenyang.

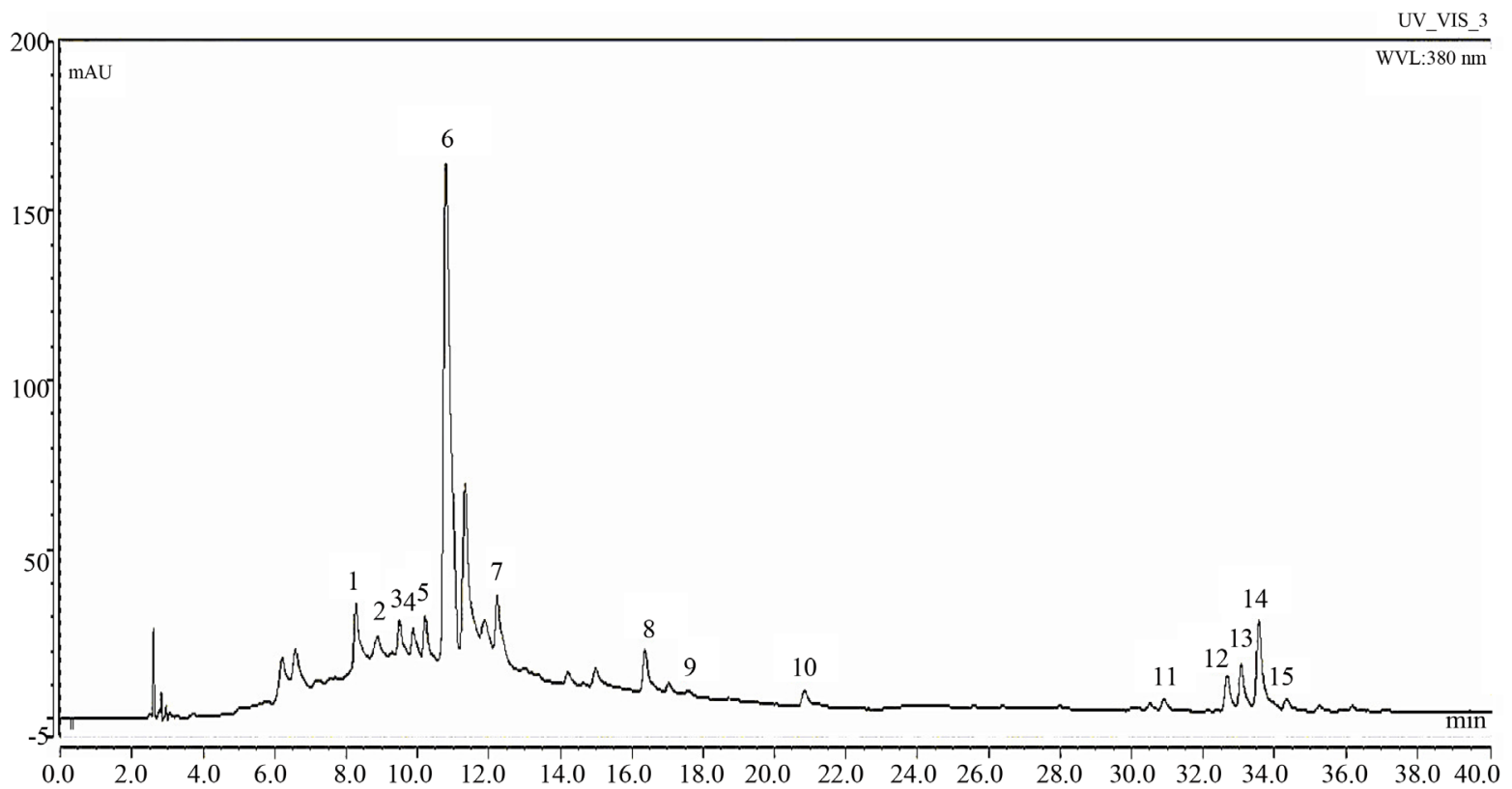

Figure 2. 15 characteristic peaks of fingerprinting. 1, 2, 3, 4, 7, 8, 11, 12, 13, 14, 15: unknown compounds, 5: rutin, 6: hyperin, 9: quercetin and 10: kaempferol.

\subsection{Quantitative Analysis of Four Active Compounds in R. chingii Hu.}

Flavonols such as rutin, hyperin, quercetin [16] and kaempferol [17] possess antioxidant activity and have been suggested to play an important role in the protective effects of berries and vegetables [18]. In the chromato- 


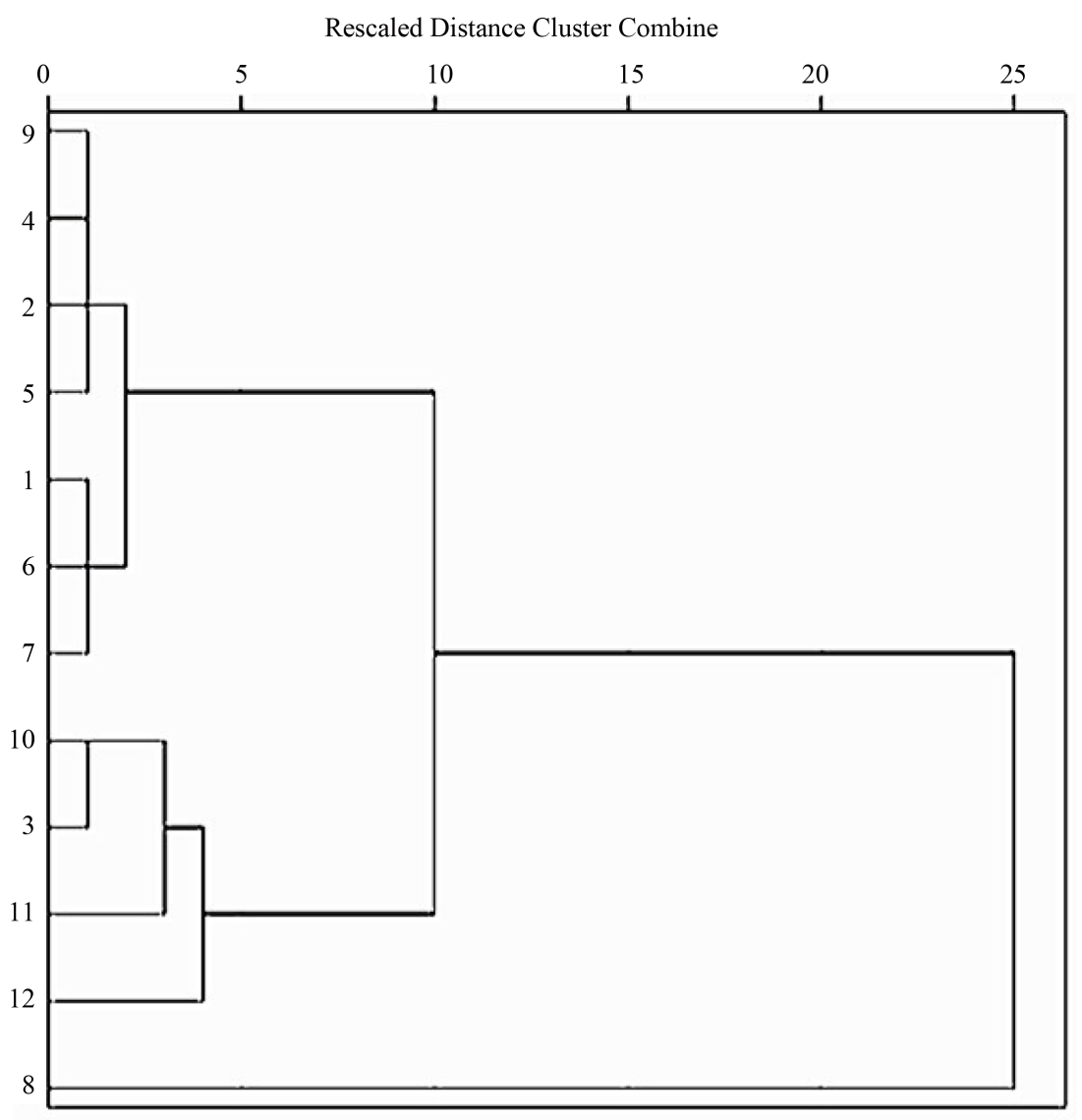

Figure 3. Dendrogram of clustering analysis. 1: Hongzhou; 2: Jinhua; 3: Zhengzhou; 4: Nanjing; 5: Guangzhou; 6: Guilin; 7: Nanning; 8: Chengdu; 9: Fuzhou; 10: Zhangzhou; 11: Changchun and 12: Shenyang.

graphic profile, four peaks (5, 6, 9 and 10) were structurally identified as rutin, hyperin, quercetin and kaempferol, respectively, by comparing their retention times (10.12 min for rutin, 10.80 for hyperin, 17.49 min for quercetin and 20.75 min for kaempferol) with those of standard substance. Therefore, the simultaneous determination of four active components in these samples was investigated by the proposed method.

\subsubsection{Calibration Curves, LOD and LLOQ}

Calibration curves were constructed by plotting the peak area (y) against the corresponding concentration of the standard solutions (x). The injection concentration, which could be detected at the signal-to-noise ratio of 3 ( $\mathrm{S} / \mathrm{N}=$ 3), was considered to be the limit of detection (LOD). The lower limit of quantification (LLOQ) was the injection concentration corresponding to the peak heights with signal-to-noise ratio of $10(\mathrm{~S} / \mathrm{N}=10)$. The detailed descriptions of the regression curves were presented in Table 5.

\subsubsection{Precision, Repeatability, Stability and Accuracy}

Intra- and inter-day precision and accuracy tests were performed by analyzing standard solutions (rutin, 18.75 $\mu \mathrm{g} \cdot \mathrm{mL}^{-1}$; hyperin, $12.50 \mu \mathrm{g} \cdot \mathrm{mL}^{-1}$; quercetin, $12.50 \mu \mathrm{g} \cdot \mathrm{mL}^{-1}$; kaempferol, $12.50 \mu \mathrm{g} \cdot \mathrm{mL}^{-1}$ ) six times a day and once a day for six sequential days, respectively. The injection repeatability was determined by the injection of continuous six times using the same sample, while the analysis repeatability was examined by the injection of six different samples, which were prepared with the same sample preparation procedure. The precision and repeatability of the solution at medium concentration were shown in Table 6. For the stability testing, the same real sample was analyzed within $24 \mathrm{~h}$ at the room temperature. The stabilities of the solution shown in RSD of retention time and peak area were all within $\pm 3 \%$ and no significant difference was observed, indicating that the solution was stable (Table 6). 
Accuracy was defined as the rate of the calculated value by the standard curve to that of its true value, expressed as recovery rate (\%). The mean recoveries and RSD for analytes at different concentrations were shown in Table 6.

\subsubsection{Determination of Four Active Compounds in Raspberry}

Under the same chromatographic conditions, four active components in raspberries were determined by the proposed method. The results were summarized in Table 7. These results indicated that the concentrations of analytes existed obvious difference between different samples, and the contents of these components could not be used as a standard of quality control, however, assessing the quality of $R$. chingii Hu. using these four active components alone is recommented to a certain extent.

Table 5. Calibration curves, LOD and LLOQ of four active components $(n=3)$.

\begin{tabular}{cccccc}
\hline Analytes & Calibration curves & $\mathrm{R}^{2}$ & Linear range $\left(\mu \mathrm{g} \cdot \mathrm{mL}^{-1}\right)$ & $\mathrm{LOD}\left(\mu \mathrm{g} \cdot \mathrm{mL}^{-1}\right)$ & $\mathrm{LLOQ}\left(\mu \mathrm{g} \cdot \mathrm{mL}^{-1}\right)$ \\
\hline rutin & $y=0.4177 x-0.3565$ & 0.9996 & $1.17-250.00$ & 0.22 & 0.73 \\
hyperin & $y=0.5932 x-0.2930$ & 0.9992 & $0.39-125.00$ & 0.07 & 0.24 \\
quercetin & $y=0.8238 x-0.3514$ & 0.9995 & $0.20-125.00$ & 0.04 & 0.12 \\
kaempferol & $y=0.6348 x-0.2658$ & 0.9993 & $0.20-125.00$ & 0.04 & 0.12 \\
\hline
\end{tabular}

Table 6. Precision, repeatability and stability of the method $(n=6)$.

\begin{tabular}{cccccc}
\hline \multirow{2}{*}{ Analytes } & $\begin{array}{c}\text { Concentration } \\
\left(\mu \mathrm{g} \cdot \mathrm{mL}^{-1}\right)\end{array}$ & Intra-day RSD (\%) & Precision & $\begin{array}{c}\text { Repeatability RSD } \\
(\%)\end{array}$ & $\begin{array}{c}\text { Stability RSD } \\
(\%)\end{array}$ \\
\cline { 3 - 6 } & 18.75 & 1.40 & 1.69 & 1.48 & 1.61 \\
Rutin & 12.50 & 0.43 & 0.32 & 0.92 & 1.01 \\
Hyperin & 12.50 & 1.05 & 0.00 & 2.88 & 2.70 \\
Quercetin & 12.50 & 2.71 & 0.51 & 2.44 & 2.92 \\
Kaempferol & & &
\end{tabular}

Table 7. Simultaneous determinations of four active components in $R$. chingii Hu. by the proposed method $(n=6)$.

\begin{tabular}{ccccc}
\hline Samples & \multicolumn{2}{c}{ Compounds $\left(\mu \mathrm{g} \cdot \mathrm{g}^{-1}\right)$} \\
\hline & Rutin & Hyperin & Quercetin & Kaempferol \\
\hline Hangzhou & $13.96 \pm 0.28$ & $181.29 \pm 0.42$ & $2.12 \pm 0.02$ & $4.00 \pm 0.06$ \\
Jinhua & $8.72 \pm 0.12$ & $478.57 \pm 1.19$ & $4.04 \pm 0.06$ & $11.69 \pm 0.15$ \\
Zhengzhou & $32.66 \pm 0.45$ & $300.03 \pm 7.25$ & $5.64 \pm 0.09$ & $7.80 \pm 0.15$ \\
Nanjing & $9.92 \pm 0.17$ & $278.60 \pm 4.41$ & $3.12 \pm 0.04$ & $6.14 \pm 0.08$ \\
Guangzhou & $18.30 \pm 0.39$ & $337.28 \pm 4.78$ & $2.73 \pm 0.05$ & $5.46 \pm 0.08$ \\
Guilin & $\mathrm{ND}$ & $508.88 \pm 3.02$ & $4.97 \pm 0.10$ & $5.68 \pm 0.11$ \\
Nanning & $9.92 \pm 0.18$ & $624.16 \pm 3.00$ & $3.57 \pm 0.04$ & $10.22 \pm 0.09$ \\
Chengdu & $40.30 \pm 0.30$ & $317.22 \pm 2.79$ & $2.70 \pm 0.05$ & $6.56 \pm 0.18$ \\
Fuzhou & $21.39 \pm 0.44$ & $418.93 \pm 1.27$ & $4.02 \pm 0.06$ & $8.75 \pm 0.09$ \\
Zhangzhou & $27.02 \pm 0.65$ & $255.34 \pm 1.11$ & $2.84 \pm 0.04$ & $5.77 \pm 0.11$ \\
Changchun & $36.28 \pm 0.69$ & $280.19 \pm 2.28$ & $1.77 \pm 0.08$ & $3.78 \pm 0.03$ \\
Shenyang & $22.40 \pm 0.29$ & $289.50 \pm 0.99$ & $2.82 \pm 0.06$ & $7.21 \pm 0.12$
\end{tabular}

ND, not detected. 


\section{Conclusion}

In this study, the total flavonoid contents and the antioxidant activities of 12 batches of raspberries (R. chingii Hu.) collected from different regions of China were investigated in detail. According to their antioxidant activities, a simple and valid chromatographic fingerprint method was developed for analysis of the sample extracts of Fupenzi by HPLC-DAD. The proposed method and 15 characteristic peaks can be used for the rapid identification and evaluation of FPZs and their differentiation from substitutes conveniently.

\section{Acknowledgements}

We gratefully acknowledge the financial support of the National Natural Science Foundation of China (U1304823).

\section{References}

[1] National Pharmacopoeia Committee (2010) Pharmacopoeia of People’s Republic of China. Part 1. Chemical Industry Press, Beijing, 359.

[2] Rommel, A. and Wrolstad, R.E. (1993) Influence of Acid and Base Hydrolysis on the Phenolic Composition of Red Raspberry Juice. Journal of Agricultural and Food Chemistry, 41, 1237-1241. http://dx.doi.org/10.1021/jf00032a014

[3] Hakkinen, S.H., Karenlampi, S.O., Heinonen, I.M., Mykkanen, H.M. and Torronen, A.R. (1998) HPLC Method for Screening of Flavonoids and Phenolic Acids in Berries. Journal of Agricultural and Food Chemistry, 77, 543-551. http://dx.doi.org/10.1002/(SICI)1097-0010(199808)77:4<543::AID-JSFA78>3.0.CO;2-I

[4] Dejaegher, B., Alaerts, G. and Matthijs, N. (2010) Methodology to Develop Liquid Chromatographic Fingerprints for the Quality Control of Herbal Medicines. Acta Chromatographica, 22, 237-258. http://dx.doi.org/10.1556/AChrom.22.2010.2.7

[5] Jiang, Y., David, B., Tu, P. and Barbin, Y. (2010) Recent Analytical Approaches in Quality Control of Traditional Chinese Medicines-A Review. Analytica Chimica Acta, 657, 9-18. http://dx.doi.org/10.1016/j.aca.2009.10.024

[6] Liang, Y., Xie, P. and Chan K. (2004) Quality Control of Herbal Medicines. Journal of Chromatography B, 812, 53-70. http://dx.doi.org/10.1016/S1570-0232(04)00676-2

[7] Gutierrez, J.R.L., Coello, J. and Maspoch, S. (2012) Enhanced Chromatographic Fingerprint of Herb Materials by Multi-Wavelength Selection and Chemometrics. Analytica Chimica Acta, 710, 40-49. http://dx.doi.org/10.1016/j.aca.2011.10.010

[8] Chou, G.X., Xu, S.J., Liu, D., Kou, G.Y., Zhang, J. and Liu, Z.J. (2009) Quantitative and Fingerprint Analyses of Chinese Sweet Tea Plant (Rubus suavissimus S. Lee). Journal of Agricultural and Food Chemistry, 57, 1076-1083. http://dx.doi.org/10.1021/jf8029397

[9] Chen, J., Lu, Y.H., Wei, D.Z. and Zhou, X.L. (2009) Establishment of a Fingerprint of Raspberries by LC. Chromatographia, 70, 981-985. http://dx.doi.org/10.1365/s10337-009-1217-6

[10] Meyers, K.J., Watkins, C.B., Pritts, M.P. and Liu, R.H. (2003) Antioxidant and Antiproliferative Activities of Strawberries. Journal of Agricultural and Food Chemistry, 51, 6887-6892. http://dx.doi.org/10.1021/jf034506n

[11] Chen, C.W. and Ho, C.T. (1995) Antioxidant Properties of Polyphenols Extracted from Green and Black Teas. Journal of Food Lipids, 2, 35-46. http://dx.doi.org/10.1111/j.1745-4522.1995.tb00028.x

[12] Re, R., Pellegrini, N., Proteggente, A., Pannala, A., Yang, M. and Rice-Evans, C. (1999) Antioxidant Activity Applying an Improved ABTS Radical Cation Decolorization Assay. Free Radical Biology and Medicine, 26, 1231-1237. http://dx.doi.org/10.1016/S0891-5849(98)00315-3

[13] Tsantili, E., Konstantinidis, K., Christopoulos, M.V. and Roussos, P.A. (2011) Total Phenolics and Flavonoids and Total Antioxidant Capacity in Pistachio (Pistachia vera L.) Nuts in Relation to Cultivars and Storage Conditions. Scientia Horticulturae, 129, 694-701. http://dx.doi.org/10.1016/j.scienta.2011.05.020

[14] Venskutonis, P.R., Dvaranauskaite, A. and Labokas, J. (2007) Raidcal Scavenging Activity and Composition of Raspberry (Rubus idaeus) Leaves from Different Locations in Lithuania. Fitoterapia, 78, 162-165. http://dx.doi.org/10.1016/j.fitote.2006.10.001

[15] Anttonen, M.J. and Karjalainen, R.O. (2005) Environmental and Genetic Variation of Phenolic Compounds in Red Raspberry. Journal of Food Composition and Analysis, 18, 759-769. http://dx.doi.org/10.1016/j.jfca.2004.11.003

[16] Makris, D.P. and Rossiter, J.T. (2001) Comparison of Quercetin and a Non-Orthohydroxy Flavonol as Antioxidants by Competing in Vitro Oxidation Reactions. Journal of Agricultural and Food Chemistry, 49, 3370-3377. http://dx.doi.org/10.1021/jf0101071 
[17] Knekt, P., Kumpulainen, J., Jarvinen, R., Rissanen, H., Heliovaara, M., Reunanen, A., Hakulinen, T. and Aromaa, A. (2002) Flavonoid Intake and Risk of Chronic Diseases. American Journal of Clinical Nutrition, 76, 560-568.

[18] Hubbard, G.P., Stevens, J.M., Cicmil, M., Sage, T., Jordan, P.A., Williams, C.M., Lovegrove, J.A. and Gibbins, J.M. (2003) Quercetin Inhibits Collagen-Stimulated Platelet Activation through Inhibition of Multiple Components of the Glycoprotein VI Signaling Pathway. Journal of Thrombosis and Haemostasis, 1, 1079-1088. http://dx.doi.org/10.1046/j.1538-7836.2003.00212.x 